\title{
A General Framework for Designing Catadioptric Imaging and Projection Systems
}

Rahul Swaminathan, Michael D. Grossberg and Shree K. Nayar

Department of Computer Science, Columbia University

New York, New York 10027

Email: \{srahul, mdog, nayar\}@cs.columbia.edu

\author{
July 2003 \\ Technical Report : CUCS-017-03
}




\section{Abstract}

New vision applications have been made possible and old ones improved through the creation and design of novel catadioptric systems. Critical to the design of catadioptric imaging is determining the shape of one or more mirrors in the system. Almost all the previously designed mirrors for catadioptric systems used case specific tools and considerable effort on the part of the designer. Recently some new general methods have been proposed to automate the design process. However, all the methods presented so far determine the mirror shape by optimizing its geometric properties, such as surface normal orientations. A more principled approach is to determine a mirror that reduces image errors.

In this paper we present a method for finding mirror shapes which meet user determined specifications while minimizing image error. We accomplish this by deriving a first order approximation of the image error. This permits us to compute the mirror shape using a linear approach that provides good results efficiently while avoiding the numerical problems associated with non-linear optimization. Since the design of mirrors can also be applied to projection systems, we also provide a method to approximate projection errors in the scene. We demonstrate our approach on various catadioptric systems and show our approach to provide much more accurate imaging characteristics. In some cases we achieved reduction in image error upto 80 percent. 


\section{Contents}

1 Design of Catadioptric Systems $r$

2 Spline-Based Mirror Design 3

2.1 Modeling the Primary Optics . . . . . . . . . . . . . . . . 4

2.2 Modeling the Mirror Shape . . . . . . . . . . . . . . . . 5 5

2.3 Mirror Normal Constraints . . . . . . . . . . . . . . . . 5

3 Reducing Scene and Image Error $\quad 6$

3.1 Relating Angular Perturbations of Tangents to Residuals . . . . . . . . . . . 7

3.2 Relating Scene Error to Residuals . . . . . . . . . . . . . . . . . . 8

3.3 Relating Image Error to Residuals . . . . . . . . . . . . . . . . . . . . . . 9

4 Computing the Mirror Shape $\quad 10$

5 Example Mirror Designs $\quad 11$

5.1 Parabolic Mirror based Single Viewpoint System . . . . . . . . . . . . . . . . . . 12

5.2 Equi-Angular Imaging System . . . . . . . . . . . . . . . . . 13

5.3 Cylindrical Panorama Imaging System . . . . . . . . . . . . . . . . 15

5.4 Skewed Plane Projection System _. . . . . . . . . . . . . 16

5.5 Conference Table Rectifying System . . . . . . . . . . . . . . . 17

5.6 Spherical Projection System . . . . . . . . . . . . . . . 19

6 Conclusions $r$ 


\section{Design of Catadioptric Systems}

The capability of a vision system is largely dependent on the type of imaging system it uses. For instance, creating 360 deg panoramic video with a single conventional (perspective) camera is impossible. It can easily be accomplished, however, with specially designed optics. Recognizing this fact, many unconventional imaging systems which use both lenses and mirrors (catadioptric systems) have been proposed in recent years.

A common use of catadioptric imaging is to enhance the field of view. Systems that achieve this goal have been developed using both planar as well as curved mirrors. Some of these systems have been designed to have a single effective viewpoint $[20,26,27,18,19,1]$. Other systems have been designed to achieve specific resolution characteristics, such as the equi-angular mapping [6], the constant-resolution mapping [9] and equi-areal mapping [16]. Systems have also been designed to acquire images of a known scene in special ways[3, 7, 2, 14, 8, 5, 17, 13].

A critical aspect of designing a catadioptric imaging system is determining the appropriate shapes of the one or more mirrors that the system uses. In general, the performance of an imaging system has two components. One is its ability to adhere to the prescribed image-to-scene map and the second is its optical image quality (determined by depth of field, coma, astigmatism, etc.[4, 12]). This paper focuses on the first of these components.

Let us consider a system that has a single mirror. To determine the shape of this mirror, the primary optics (perspective lens, telecentric lens or otherwise) and the image-to-scene map (a map from pixels in the image to scene points or viewing directions) need to be known. Hicks and Perline [15] were the first to pose the problem for mirror design using "geometric distributions".

The mirror for each of the systems designed previously was found by using the constraints imposed by the image-to-scene map to derive partial differential equations (PDEs) that the mirror must satisfy. The mirror shape was then determined by finding solutions to the PDEs. This approach requires considerable skill and effort on the part of the designer. A more general approach using splines to model and compute the mirror shape was recently presented in [25]. This method is similar to an earlier method developed in [11] to recover the shape of a specular surface given known scene points and a perspective camera.

An important problem that arises in designing mirrors is that there may not exist a single mirror that can exactly achieve the required image-to-scene map. In such cases, all the mirror design methods described above determine the "best-fit" mirror surface that minimizes errors in its three dimensional shape (surface normals of the mirror). However, as shown in Fig.1, a mirror that 


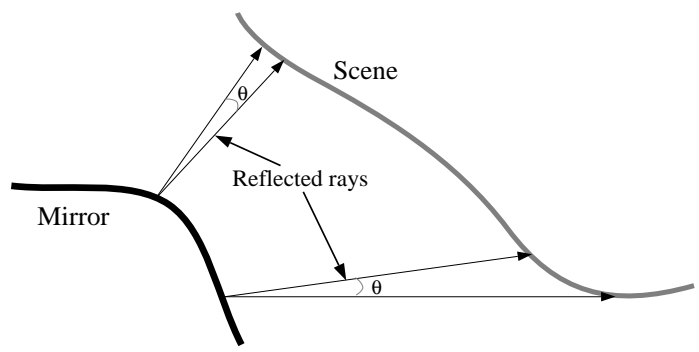

Figure 1: Two points on a mirror of a catadioptric system reflecting rays towards the scene are perturbed by the same small angle $\theta$. Clearly the two equal perturbations cause unequal errors on the scene projection. Most mirror design methods try to minimize errors in the shape of the mirror itself (such as surface normal orientation) without regard to its effects on the scene or the acquired image. Thus, the image acquired with such a mirror is not guaranteed to exhibit minimum image error.

minimizes errors in its normals need not minimize image errors. That is, the resulting mirror may have normals that are close to the desired set of normals but produces very large distortions in the captured image. Since we are interested in the geometric quality of the image captured by the imaging system, the correct approach is to find a mirror that minimizes image errors.

Note that a method for designing mirrors for imaging can also be used to design projection systems. In this case, the mirror must minimize scene projection errors instead. In general, minimizing either the image error or scene error requires a non-linear optimization, which can be highly unstable. In [25], the authors recognized this problem and addressed it by using a heuristic weighting approach. However, this technique does not accurately model either image or scene errors and hence does not guarantee good solutions.

In this work, we derive first-order approximations to image and scene errors. We incorporate these approximations into the algorithm in [25] to determine the mirror shape that minimizes image or scene errors, without the need for a costly non-linear optimization. We present experimental results to demonstrate the effectiveness of such an approach.

\section{Spline-Based Mirror Design}

The catadioptric system to be designed is assumed to consist of some known primary optics and an unknown mirror. The primary optics are assumed to be fixed and known. The designer of the imaging or projection system presents the requirements of the system in terms of a map from pixels in the image to points in the scene. We call this the image-to-scene map which we denote 


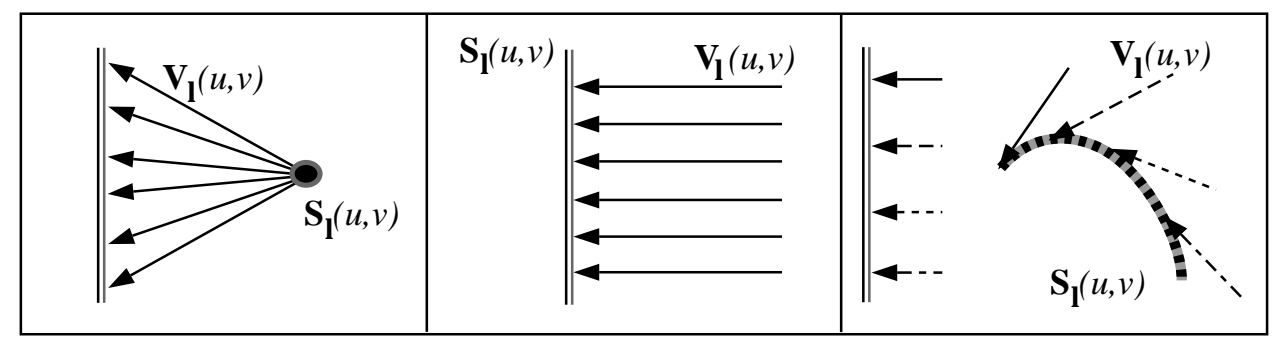
(a) Perspective
(b) Orthographic
(c) Generalized

Figure 2: The different models of the primary optics for which our method can compute a mirror shape. (a) A perspective imaging model found in most projectors and imaging systems. (b) Orthographic projection, typically obtained with telecentric lenses. (c) The generalized imaging model (caustic model) , wherein each pixel can have its own associated viewpoint and viewing direction. This last model is most general and allows the most flexibility in designing catadioptric systems.

$\mathcal{M}(u, v)$. Our goal is the find the mirror which along with the known primary optics implements the desired image-to-scene map $\mathcal{M}$.

In this paper we use a previously proposed method for general imaging system design using splines [25]. Our emphasis in this work however, is to modify the equations used in previous work so that the mirror we compute minimizes image or scene errors. The previous approach based on a specular shape recovery technique [11] essentially determines the mirror by minimizing errors in its surface normal orientations. Before we present the details of our approach, we shall introduce certain notation from previous work which will be useful. Throughout this paper we will address the problem of catadioptric imaging system design. However, the same analysis can be directly applied to projector design as well.

\subsection{Modeling the Primary Optics}

The primary optics of the catadioptric system could either use a conventional lens (perspective projection) as in Fig. 2(a), or a telecentric lens (orthographic projection) as shown in Fig. 2(b), or the generalized model[10, 23] (see Fig. 2(c)). Referring to Fig. 2(c)), in general a pixel $(u, v)$ in the image possesses a viewpoint $\mathbf{S}_{\mathbf{l}}(u, v)$ and a viewing direction $\mathbf{V}_{\mathbf{l}}(u, v)$. For a perspective lens, the viewpoints $\mathbf{S}_{1}(u, v)$ collapse to a single point. 


\subsection{Modeling the Mirror Shape}

We describe the mirror shape $\mathbf{S}_{\mathbf{r}}$ in terms of the model for the primary optics as:

$$
\mathbf{S}_{\mathbf{r}}(u, v)=\mathbf{S}_{\mathbf{l}}(u, v)-D(u, v) \mathbf{V}_{\mathbf{l}}(u, v),
$$

where $D(u, v)$ is the distance of the mirror from the viewpoint surface, modeled using the tensor product spline:

$$
D(u, v)=\sum_{i=1}^{K_{f}} \sum_{j=1}^{K_{g}} c_{i, j} f_{i}(u) g_{j}(v),
$$

where $f_{i}(u)$ and $g_{j}(v)$ are 1-D spline basis functions, $c_{i, j}$ are the coefficients of the spline model, and $K_{f} \cdot K_{g}$ are the number of spline coefficients.

\subsection{Mirror Normal Constraints}

Fig. 3 shows a catadioptric system used to image some known scene. The mirror surface $\mathbf{S}_{\mathbf{r}}(u, v)$ must implement the user provided image to scene mapping $\mathcal{M}$ by reflecting each scene point $\mathcal{M}(u, v)$ along the scene ray $\mathbf{V}_{\mathbf{r}}(u, v)$ into the primary optics, where:

$$
\mathbf{V}_{\mathbf{r}}(u, v)=\frac{\mathbf{S}_{\mathbf{r}}(u, v)-\mathcal{M}(u, v)}{\left|\mathbf{S}_{\mathbf{r}}(u, v)-\mathcal{M}(u, v)\right|} .
$$

This constrains the surface normal of the mirror $\mathbf{N}_{\mathbf{r}}$ as:

$$
\mathbf{N}_{\mathbf{r}}(u, v)=\frac{\mathbf{V}_{\mathbf{l}}(u, v)-\mathbf{V}_{\mathbf{r}}(u, v)}{\left|\mathbf{V}_{\mathbf{l}}(u, v)-\mathbf{V}_{\mathbf{r}}(u, v)\right|} .
$$

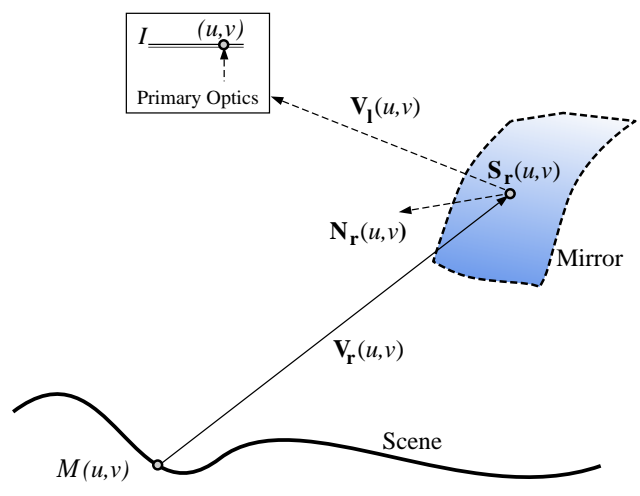

Figure 3: A catadioptric imaging system consisting of some known primary optics and a mirror. In general, a pixel $(u, v)$ in the image maps to the scene point $\mathcal{M}(u, v)$ after reflecting at $\mathbf{S}_{\mathbf{r}}(u, v)$ on the mirror. This forces constraints on the surface normals $\mathbf{N}_{\mathbf{r}}(u, v)$ of the mirror. 
The tangent vectors $\mathbf{T}_{u}(u, v)=\frac{\partial \mathbf{S}_{\mathbf{r}}(u, v)}{\partial u}$ and $\mathbf{T}_{v}(u, v)=\frac{\partial \mathbf{S}_{\mathbf{r}}(u, v)}{\partial v}$ on the mirror surface must be orthogonal to the surface normal in Eq.(4). This provides two constraints on the mirror shape:

$$
\begin{aligned}
& \mathbf{T}_{u}(u, v) \cdot \mathbf{N}_{\mathbf{r}}(u, v)=0, \\
& \mathbf{T}_{v}(u, v) \cdot \mathbf{N}_{\mathbf{r}}(u, v)=0 .
\end{aligned}
$$

Rearranging the terms and substituting Eq.(1) in Eq.(5), we get:

$$
\begin{aligned}
& \left(\frac{\partial D}{\partial u} \mathbf{V}_{\mathbf{l}}+D \frac{\partial \mathbf{V}_{\mathbf{l}}}{\partial u}-\frac{\partial \mathbf{S}_{1}}{\partial u}\right) \cdot \mathbf{N}_{\mathbf{r}}=0 \\
& \left(\frac{\partial D}{\partial v} \mathbf{V}_{1}+D \frac{\partial \mathbf{V}_{1}}{\partial v}-\frac{\partial \mathbf{S}_{1}}{\partial v}\right) \cdot \mathbf{N}_{\mathbf{r}}=0 .
\end{aligned}
$$

Now, substituting Eq.(2) in Eq.(7), we get:

$$
\begin{aligned}
& \left(\mathbf{V}_{\mathbf{1}} \sum_{i, j} c_{i, j} f_{i}^{\prime}(u) g_{j}(v)+\frac{\partial \mathbf{V}_{\mathbf{1}}}{\partial u} \sum_{i, j} c_{i, j} f_{i}(u) g_{j}(v)-\frac{\partial \mathbf{S}_{\mathbf{1}}}{\partial u}\right) \cdot \mathbf{N}_{\mathbf{r}}=0 \\
& \left(\mathbf{V}_{\mathbf{1}} \sum_{i, j} c_{i, j} f_{i}(u) g_{j}^{\prime}(v)+\frac{\partial \mathbf{V}_{\mathbf{1}}}{\partial v} \sum_{i, j} c_{i, j} f_{i}(u) g_{j}(v)-\frac{\partial \mathbf{S}_{\mathbf{1}}}{\partial v}\right) \cdot \mathbf{N}_{\mathbf{r}}=0 .
\end{aligned}
$$

where $f_{i}^{\prime}(u)$ and $g_{j}^{\prime}(v)$ denote the derivatives of $f_{i}(u)$ and $g_{j}(v)$, respectively. Note that the above constraints quantify the error in mirror shape in terms of the dot product error between the tangents and the desired surface normals of the mirror. Furthermore they are linear in the spline coefficients $c_{i, j}$.

\section{Reducing Scene and Image Error}

We now present a simple technique to reformulate the constraints in Eqs. (5-8) so that solutions to the modified equations will approximately minimize image or scene errors. Before doing so however, we need to define scene error and image errors.

Consider a catadioptric system that must implement some image-to-scene map $\mathcal{M}(u, v)$. When no mirror shape exists that can exactly implement $\mathcal{M}(u, v)$, the mirror shape is approximate. Therefore a light ray corresponding to pixel $(u, v)$ gets reflected towards a scene point other than the required point $\mathcal{M}(u, v)$. Let $\eta(u, v)$ be the effective (possibly erroneous) image-to-scene map 
that the catadioptric system with a given mirror implements. The scene error $\xi_{\rho}$ at a point $(u, v)$ is then given by:

$$
\xi_{\rho}=|\eta(u, v)-\mathcal{M}(u, v)|
$$

For an imaging system, the image error can be computed by simply inverse mapping the effective scene point $\eta(u, v)$ onto the image using the ideal image-to-scene map $\mathcal{M}(u, v)$. The image error $\xi_{I}$ at pixel $(u, v)$ is:

$$
\xi_{I}=\left|\mathcal{M}^{-1}(\eta(u, v))-(u, v)\right|
$$

When an exact mirror shape exists, the errors $\xi_{I}, \xi_{\rho}$ and Eqs.(5) all vanish. When no mirror shape exists that implements the desired image-to-scene map, the Eqs. (5) are not satisfied and can be rewritten as:

$$
\xi_{O}=\left(\begin{array}{c}
\xi_{O, u} \\
\xi_{O, v}
\end{array}\right)=\left(\begin{array}{c}
\mathbf{T}_{u} \cdot \mathbf{N}_{\mathbf{r}} \\
\mathbf{T}_{v} \cdot \mathbf{N}_{\mathbf{r}}
\end{array}\right)
$$

where $\xi_{O, u}$ and $\xi_{O, v}$ denote the residues corresponding to the two constraint equations respectively. The least squares solution of Eqs. (5) minimizes the mean square of the residual $\xi_{O}$. Our goal is to formulate linear equations whose least square solutions better approximate the image or scene errors. To do so, we first derive a relationship between the residual and angular perturbations of the tangent vectors. Next we relate the angular tangent perturbations to errors in the reflected rays and thus scene errors. The last step is to relate the scene errors to image errors.

\subsection{Relating Angular Perturbations of Tangents to Residuals}

Referring to Eq. (1) for the mirror shape, the surface tangents are given by $\mathbf{T}_{u}=\frac{\partial \mathbf{S}_{\mathbf{r}}}{\partial u}$ and $\mathbf{T}_{v}=\frac{\partial \mathbf{S}_{\mathbf{l}}}{\partial u}$ respectively. Taking tangent $\mathbf{T}_{u}$ into consideration, we first note that it can be rewritten as:

$$
\mathbf{T}_{u}=\frac{\partial D}{\partial u} \mathbf{V}_{1}+\left(D \frac{\partial \mathbf{V}_{1}}{\partial u}-\frac{\partial \mathbf{S}_{1}}{\partial u}\right)
$$

For a given pixel $(u, v)$, the quantities $\frac{\partial \mathbf{V}_{\mathbf{1}}}{\partial u}$ and $\frac{\partial \mathbf{S}_{\mathbf{1}}}{\partial u}$ depend on the primary optics and are independent of the mirror shape (tensor-spline coefficients). In analyzing the perturbations of the tangents we consider the location of a point on the mirror $\mathbf{S}_{\mathbf{r}}(u, v)$ to be essentially fixed. Thus, $D(u, v)$ changes slowly with respect to changes in the spline coefficients. The variation in tangents with respect to the coefficients is thus almost entirely due to changes in $\frac{\partial D(u, v)}{\partial u}$. This restricts $\mathbf{T}_{u}$ to a plane $\mathcal{P}_{u}$ spanned by the vectors $\mathbf{V}_{1}$ and $D \frac{\partial \mathbf{V}_{\mathbf{l}}}{\partial u}-\frac{\partial \mathbf{S}_{1}}{\partial u}$. Thus, perturbations to $\mathbf{T}_{u}$ can be expressed simply as a rotation in this plane. 
Let $\pi_{u}\left(\mathbf{N}_{\mathbf{r}}\right)$ be the projection of $\mathbf{N}_{\mathbf{r}}$ onto the plane $\mathcal{P}_{u}$. We note that the component of $\mathbf{N}_{\mathbf{r}}$ which is orthogonal to plane $\mathcal{P}_{u}$ is automatically orthogonal to $\mathbf{T}_{u}$. Therefore this component of $\mathbf{N}_{\mathbf{r}}$ is irrelevant. We can then express the angle $\theta_{u}$ between $\pi_{u}\left(\mathbf{N}_{\mathbf{r}}\right)$ and $\mathbf{T}_{u}$ in terms of the residual as:

$$
\cos \theta_{u}=\xi_{O, u} /\left(\mid \mathbf{T}_{u} \| \pi_{u}\left(\mathbf{N}_{\mathbf{r}}\right)\right)
$$

Similarly, $\xi_{O, v}$ can be related to $\theta_{v}$ as

$$
\cos \theta_{v}=\xi_{O, v} /\left(\mid \mathbf{T}_{v} \| \pi_{v}\left(\mathbf{N}_{\mathbf{r}}\right)\right)
$$

The angles $\left(\theta_{u}, \theta_{v}\right)$ specify the directions of the tangents for a given iteration. We write the first order approximation for a perturbation of these angles by $\left(\Delta \theta_{u}, \Delta \theta_{v}\right)$ as:

$$
\left(\begin{array}{c}
\Delta \theta_{u} \\
\Delta \theta_{v}
\end{array}\right) \approx\left(\begin{array}{c}
\cot \left(\theta_{u}\right) \\
\cot \left(\theta_{v}\right)
\end{array}\right)-L\left(\begin{array}{c}
\xi_{O, u} \\
\xi_{O, v}
\end{array}\right)
$$

where $L$ is a diagonal matrix with diagonal elements

$$
\begin{aligned}
& L_{1}=-1 / \sin \left(\theta_{u}\right)\left|\mathbf{T}_{u}\right|\left|\pi_{\alpha_{u}}\left(\mathbf{N}_{\mathbf{r}}\right)\right| \\
& L_{2}=-1 / \sin \left(\theta_{v}\right)\left|\mathbf{T}_{v}\right|\left|\pi_{\alpha_{v}}\left(\mathbf{N}_{\mathbf{r}}\right)\right|
\end{aligned}
$$

The quantity $\pi_{u}\left(\mathbf{N}_{\mathbf{r}}\right)$ can be computed at every iteration of our method and does not depend on the unknown tangents. The length $\left|\mathbf{T}_{u}\right|$ does depend on the unknown tangents. However, in our iterative method to determine the mirror shape, we find that $\left|\mathbf{T}_{u}\right|$ remains relative constant across iterations. The reasons for this can be seen in Eq. (11). As already noted, $\mathbf{T}_{u}$ is a sum of three terms with $\frac{\partial \mathbf{S}_{\mathbf{I}}}{\partial u}$ constant for each iteration and with $D \frac{\partial \mathbf{V}_{\mathbf{l}}}{\partial u}$ changing slowly because it depends only on the mirror position.

So far we derived a first order relationship between the residuals of the constrains of Eqs. (5) and perturbations of the tangent vectors. We shall use this relationship next to determine the scene errors.

\subsection{Relating Scene Error to Residuals}

We now present our method of relating tangent perturbations to scene errors. This will be used to modify Eqs. (5) in order to approximate the scene errors.

The three dimensional vector which represents the scene error is:

$$
\xi_{\rho}=\left(\xi_{\rho, x}, \xi_{\rho, y}, \xi_{\rho, z}\right)=\eta(u, v)-M(u, v)
$$


For a given pixel we consider the vector scene error $\xi_{\rho}$ as a function of the angles $\theta_{u}$ and $\theta_{v}$. The first order approximation of the vector scene error due to angular perturbations $\Delta \theta_{u}$ and $\Delta \theta_{v}$ is then given by:

$$
\left(\begin{array}{c}
\xi_{\rho, x}\left(\theta_{u}, \theta_{v}\right) \\
\xi_{\rho, y}\left(\theta_{u}, \theta_{v}\right) \\
\xi_{\rho, z}\left(\theta_{u}, \theta_{v}\right)
\end{array}\right)+J\left(\xi_{\rho}\right)\left(\begin{array}{c}
\Delta \theta_{u} \\
\Delta \theta_{v}
\end{array}\right)
$$

where $J\left(\xi_{\rho}\right)$ is the Jacobian, given by:

$$
J\left(\xi_{\rho}\right)=\left(\begin{array}{cc}
\frac{\partial \xi_{\rho, x}}{\partial u} & \frac{\partial \xi_{\rho, x}}{\partial v} \\
\frac{\partial \xi_{\rho, y}}{\partial u} & \frac{\partial \xi_{\rho, y}}{\partial v} \\
\frac{\partial \xi_{\rho, z}}{\partial u} & \frac{\partial \xi_{\rho, z}}{\partial v}
\end{array}\right) .
$$

When the angles of the tangent vectors of the mirror $\mathbf{T}_{u}$ and $\mathbf{T}_{v}$ are perturbed by a small amount $\Delta \theta_{u}$ and $\Delta \theta_{v}$ about $\pi / 2$, the cotangent term in Eq. (14) vanishes and $\sin (\pi / 2)=1$ in Eq. (15). Combining these equations with Eq. (17) we have the first order approximation for scene error as

$$
\xi_{\rho} \approx J\left(\xi_{\rho}\right) L \xi_{O}
$$

Observe from Eq. (5) that the residuals $\xi_{O}$ are given by a pair of linear equations in the spline coefficients for each pixel. We multiply each pair of equations from the left by the $3 \times 2$ matrix $J\left(\xi_{\rho}\right) L$ to obtain three linear equations in the spline coefficients. A least squares solution of these modified linear equations approximates the minimization of the scene error to first order. These are the equations we use to compute the mirror shape for catadioptric projection systems.

\subsection{Relating Image Error to Residuals}

For imaging systems we wish to minimize the image error defined in Eq. (9). This can be achieved by mapping the scene errors back to the image using the inverse $\mathcal{M}^{-1}$ of the image-to-scene map. However, since the Jacobian of the image-to-scene map $J(\mathcal{M})$ is a $3 \times 2$ matrix, we employ a left pseudo-inverse to transform the scene error to the image error as

$$
\xi_{I} \approx\left(J(\mathcal{M})^{T} J(\mathcal{M})\right)^{-1} J(\mathcal{M})^{T} J\left(\xi_{\rho}\right) \xi_{\rho}
$$

Combining this with Eq. (19), a first order approximation for image error is then:

$$
\xi_{I} \approx\left(\left(J(\mathcal{M})^{T} J(\mathcal{M})\right)^{-1} J(\mathcal{M})^{T} J\left(\xi_{\rho}\right) L \xi_{O}\right.
$$


Hence, we now have a simple method to transform the residuals in Eqs. (5) into the image or scene error. To do so, we simply multiply the constraint equations by an appropriate transformation matrix as in Eqs. $(19,21)$. This results in a new set of linear equations that approximate, to first order, the minimization of the scene or image error respectively.

\section{Computing the Mirror Shape}

We now present an iterative algorithm to compute the mirror shape for a desired catadioptric system using the modified constraints presented in the previous section.

We first note that every pixel provides two constraint equations which are linear in the spline coefficients. Refering to Eq.2, we have $K_{f} \cdot K_{g}$ spline coefficients that need to be estimated. We

therfore need to use constraints at least $\frac{K_{f} K_{g}}{2}$ pixels to linearly solve for the coefficients. For accurate mirror shape computation we typically use every image pixel. We then estimate spline coefficients by solving an overdetermined system of equations formed by stacking together all the constraints.

It should be noted that to solving for the mirror shape requires knowing the mirror surface normal at every sampled image pixel. Referring to Fig. 4, we observe that the mirror can lie anywhere within the field of view of the primary optics. The two mirrors $\mathbf{S}_{\mathbf{r}}{ }^{(1)}$ and $\mathbf{S}_{\mathbf{r}}{ }^{(2)}$ in Fig. 4 lie at different locations and must reflect the same scene point $\mathcal{M}(u, v)$ into the primary optics. The direction $\mathbf{V}_{\mathbf{r}}(u, v)$ along which the scene point $\mathcal{M}(u, v)$ is viewed depends on the mirror location. This in turn influences the surface normals on the mirror and hence its shape.

We resolve this cyclical dependency using an iterative method. We first approximate the mirror by a set of facets (say, one for each pixel) that lie on a plane. Only the distance of the plane from the primary optics is chosen by the designer. We then estimate the initial set of surface normals using the reflected viewing direction $\mathbf{V}_{\mathbf{r}}$ and incident ray from the primary optics $\mathbf{V}_{\mathbf{l}}$. These normals are used to linearly solve for the mirror shape. We now iterate by using the computed mirror shape in each iteration to estimate a better estimate of the surface normals for the next iteration. Typically, convergence is achieved within 10 iterations. An example of how the mirror shape evolves from the initial planar guess to its final shape is shown in Fig. 5. In most cases, the mirror shape is close to its final shape after just one iteration.

Note that the iterative approach is required only for finte depth scenes. When the scene can be assumed to be very distant (at infinity), only viewing directions need to be specified. In this 


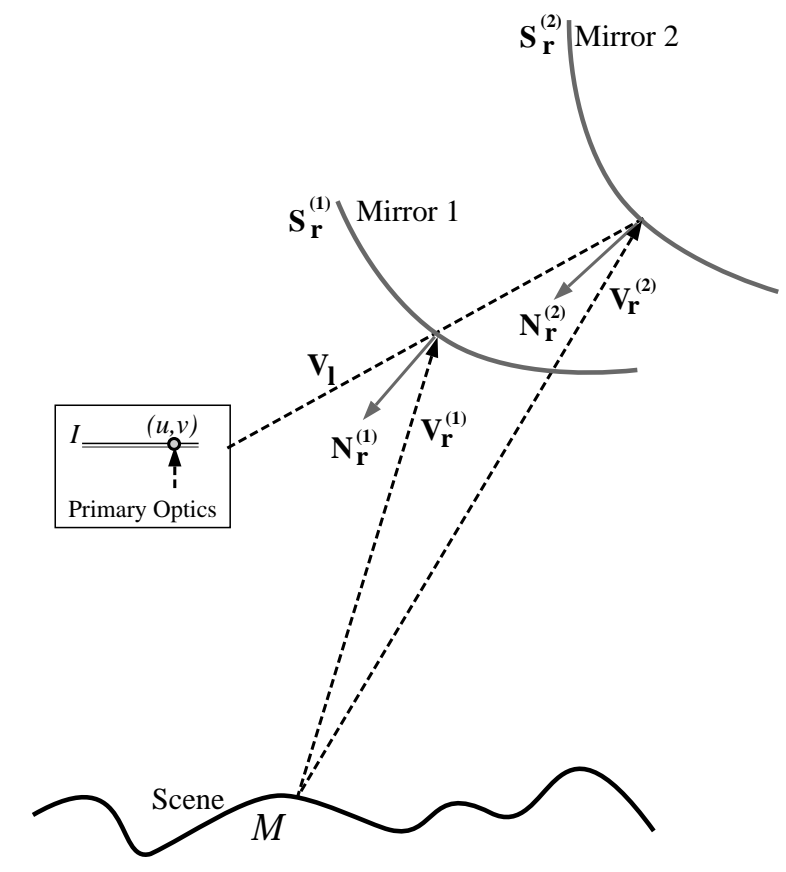

Figure 4: The direction along which a prescribed scene point $\mathcal{M}(u, v)$ is viewed by the sensor depends on the position of the point of reflection on the mirror. This is particularly true for close by scenes rather than distant scenes. As shown here, changing the location from $\mathbf{S}^{(0)}$ to $\mathbf{S}^{(1)}$ alters the reflection direction, thus changing the surface normal.

setting the required mirror's surface normals do not change since the incident rays (from primary optics) and reflected rays (viewing directions) remain constant, and the mirror shape is computed directly in one iteration alone.

\section{$5 \quad$ Example Mirror Designs}

We now use our method to compute mirror shapes for different catadioptric imaging and projection systems. These include previously designed systems as well as two new ones. The previously designed systems we present include the parabolic reflector based single viewpoint system [19, 1], the equi-angular system [6], the plane rectifying system [14]. We also present a recently proposed panoramic imaging system [13].The new systems we present include an imaging system for teleconferencing applications and a catadioptric spherical emmersive display projector design. Specially in the last two cases, no mirror exists that can provide the required image warping. We compare mirror shapes computed using the traditional mirror shape based approach to our 


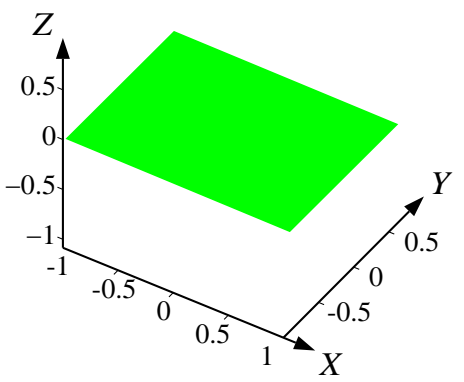

Initial guess (planar)

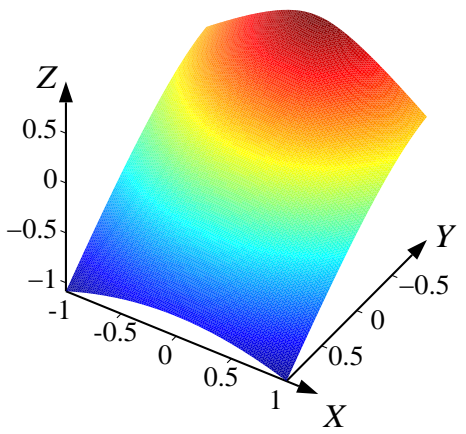

Iteration: 5

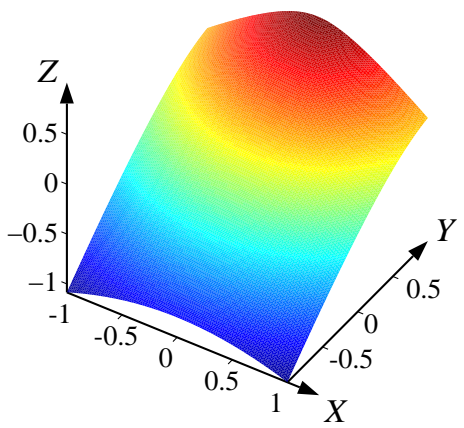

Iteration: 1

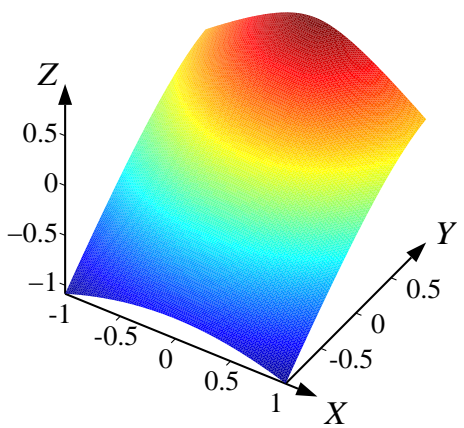

Iteration: 10 (Final iteration)

Figure 5: Convergence of the mirror shape designed for the skewed plane projection in Fig. 9. (a) Initial planar guess for the mirror with facets having different surface normals. (b) After the first iteration, the mirror shape is already close to the final solution. (c) After 5 iterations, the mirror changes a negligible amount indicating fast convergence. (d) The final mirror shape obtained after 10 iterations.

image error minimizing approach. We also present the viewpoint loci (cautsics) for the computed catadioptric systems. A detailed explanation of computing caustics for the spline based mirrors can be found in [24].

\subsection{Parabolic Mirror based Single Viewpoint System}

We computed the mirror for a para-catadioptric [19] imaging system using a telecentric lens for the primary optics (orthographic projection). The theoretical mirror shape for this system is known to be parabolic. The mirror was designed by specifying an image-to-scene map such that all the imaged rays pass through a virtual viewpoint located 1 inch below the apex of the reflector.

As seen in Fig. 6(a), the profile of the computed mirror agrees with the analytic parabolic profile. We show the three-dimensional shape of the computed mirror in Fig. 6(b). The errors in the 


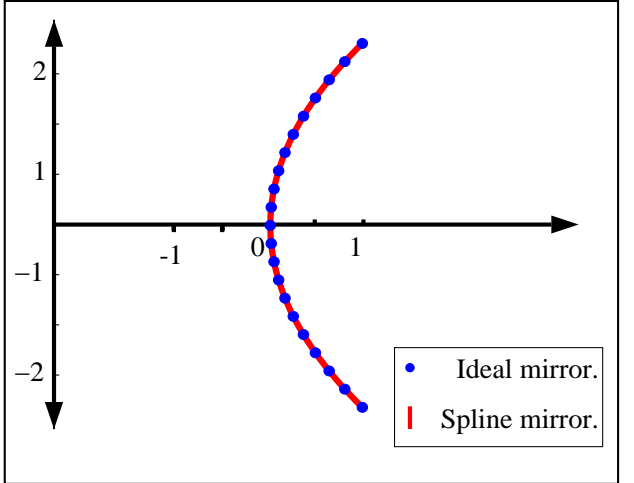

(a) Comparison between computed and ideal mirror

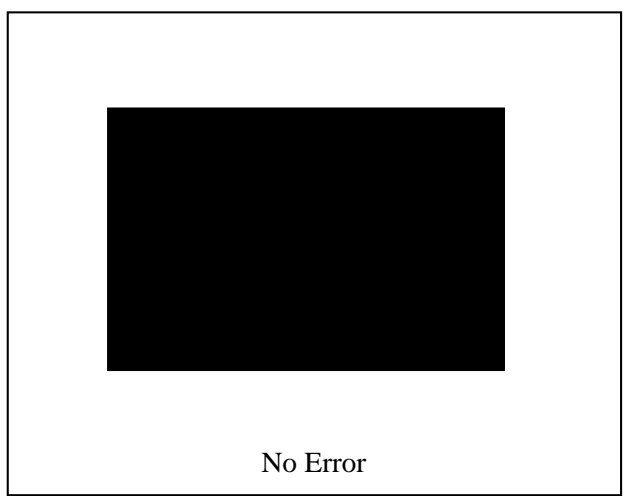

(c) Image errors (view angle) in degrees

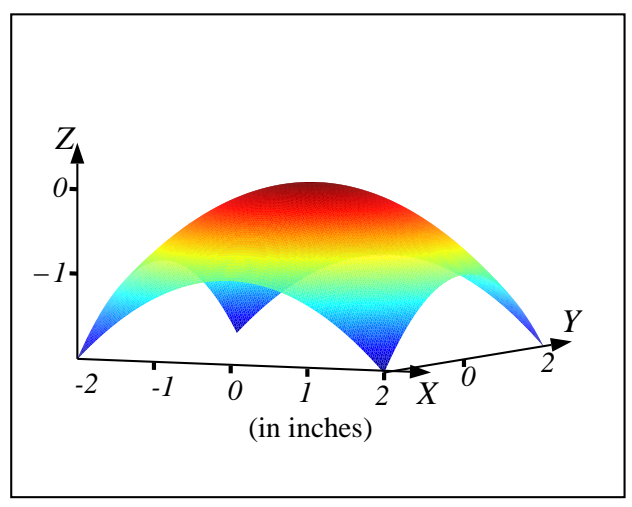

(b) Computed mirror in 3D

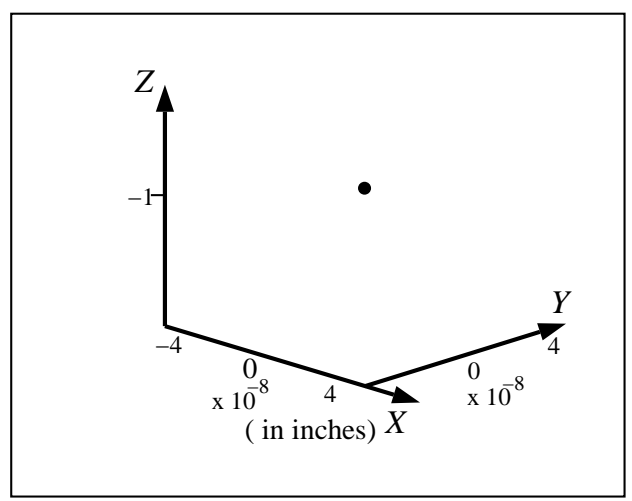

(d) Caustic of the imaging system

Figure 6: Results of applying the mirror design method for the para-catadioptric imaging system [19]. (a) The mirror shape computed using the proposed method is identical to thlo le ground truth solution (parabolic). (b) The 3D shape of the mirror shown for visualization. (c) The mirror design being exactly equal to the ground truth produced no image projection error. (d) The caustic is a very compact point cloud (single viewpoint), as expected.

recovered mirror shape, in terms of the viewing angles, are practically zero. The small errors we observed (on the order of $10^{-8}$ ) were due to numerical imprecision. Finally, the caustic surface (viewpoint locus) for this system was computed and found to be a very compact cluster of points (essentially a single viewpoint, as expected).

\subsection{Equi-Angular Imaging System}

We now present results for the equi-angular imaging system developed by Chahl and Srinivasan [6]. In [6], a family of mirror surfaces that maintain a linear map between scene rays and reflected 


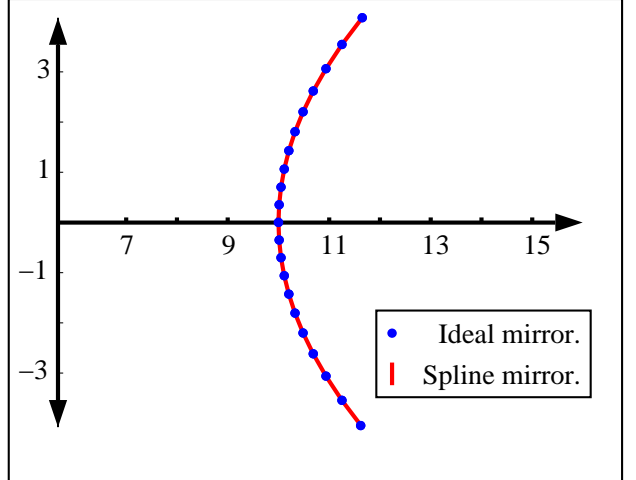

(a) Comparison between computed and ideal mirror

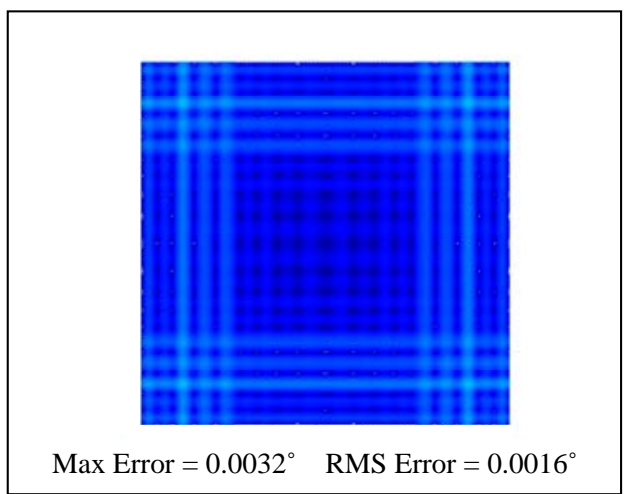

(c) Image errors (view angle) in degrees

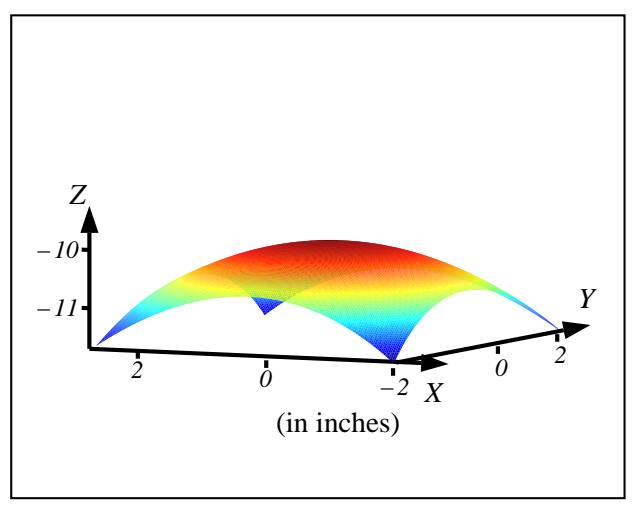

(b) Computed mirror in 3D

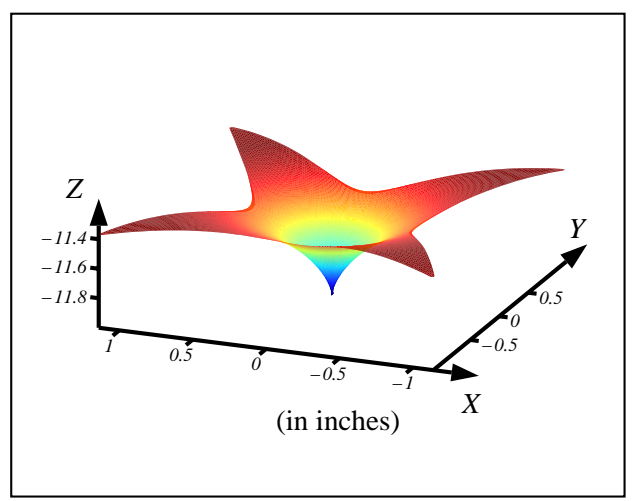

(d) Caustic of the imaging system

Figure 7: Results of applying the proposed mirror design method for the equi-angular imaging system [6]. (a) The computed mirror shape is identical to the ground truth (see Eq.(23)). (b) The three dimensional shape of the computed mirror. (c) The mirror design being an close fit to ground truth, produces negligible viewing direction errors. (d) The computed caustic surface.

rays entering the primary optics were derived as:

$$
\cos (\theta(1+\beta) / 2)=\left(r / r_{0}\right)^{-(1+\beta) / 2}
$$

where, $r^{2}=x^{2}+y^{2}, \theta=\tan ^{-1}(y / x)$ and $\beta$ controls the angular magnification.

We tested our method using various values of $\beta(\beta=3,5,7)$. In all cases the computed mirror shape was identical to ground truth. We present results for the case $\beta=5$, for which the mirror profile (ground truth) is given by:

$$
x\left(x^{2}-3 y^{2}\right)=x_{0}^{3} .
$$

As shown in Fig. 7(a), the plot of the computed mirror closely matches the ground truth shape (see Eq.(23)). The errors in viewing direction (scene ray) across the image plotted in degrees are negligible as seen in Fig. $7(\mathrm{c})$. The regular pattern in the error plot is possibly due to the 


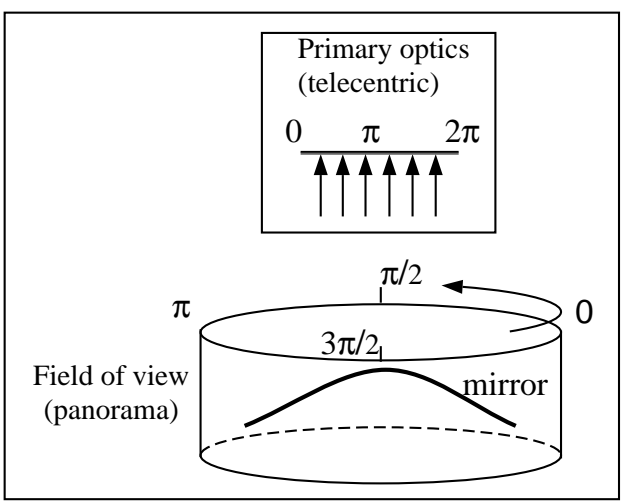

(a) Setup for cylindrical panorama system

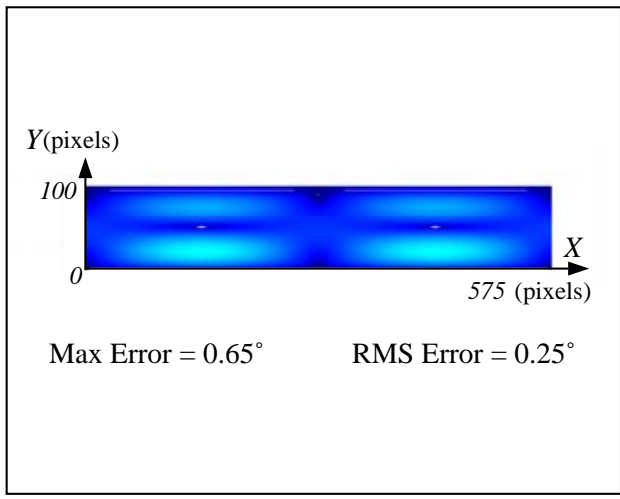

(c) Image errors (view angle) in degrees

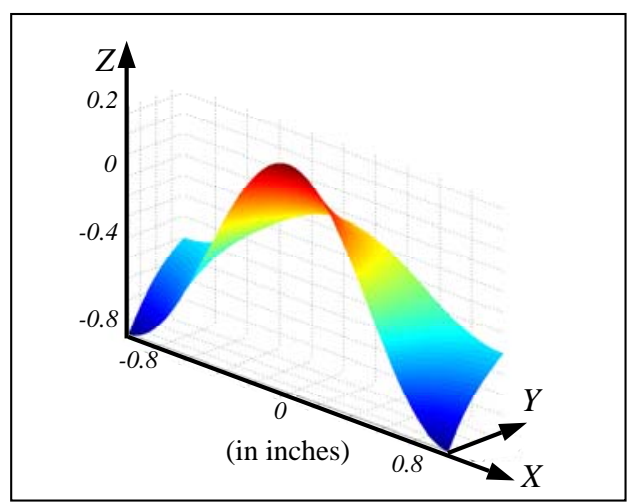

(b) Computed mirror in 3D

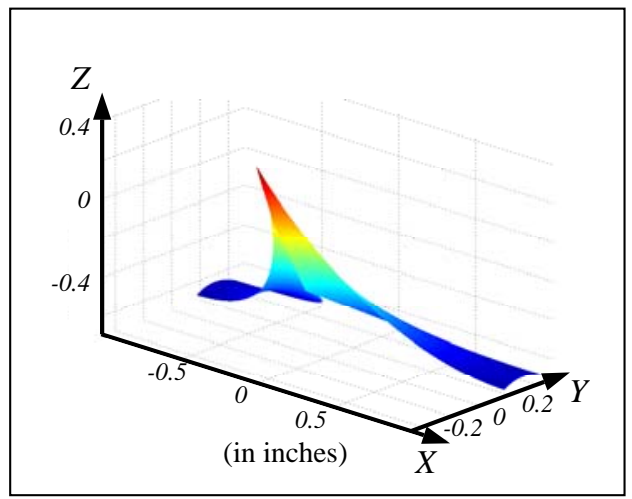

(d) Caustic of the imaging system

Figure 8: Results for the cylindrical panoramic imaging system [13, 21]. (a) The setup shows a mirror reflecting the scene around the system into the primary optics to directly form a cylindrical panorama in the image. (b) The computed mirror shape closely resembles those obtained previously [13]. (c) The computed mirror exhibits small errors in the viewing directions across the image. (d) Again, the computed caustic of this complex catadioptric system was not known until now.

piece-wise nature of the spline model, used for the mirror. This catadioptric system does not have a single viewpoint. The computed caustic surface is shown in Fig. 7(d).

\subsection{Cylindrical Panorama Imaging System}

Most omnidirectional catadioptric cameras acquire a panoramic image that is distorted and requires computational resources to unwarp into a cylindrical panorama. Hicks [13] and Srinivasan [21] independently presented a design of a catadioptric camera that directly acquires a cylindrical panoramic image. Here, we design the same system using our method.

Fig. 8(a) shows a schematic of the desired imaging system. A mirror reflects the scene into the 
primary optics consisting of a telecentric lens to form a cylindrical panorama in the image. Each image pixel $(u, v)$ is mapped directly to the desired viewing direction $\mathbf{V}_{\mathbf{r}}(u, v)$ (assuming the scene to be at infinity). The horizontal field of view is 360 degrees, while the vertical field of view is 60 degrees.

Since the scene is assumed to lie at infinity, it is possible to compute the mirror shape using a single iteration of our method. We computed the mirror, shown in Fig. 8(b), for an image of aspect ratio $575: 100$. Note that, only for certain aspect ratios of the image does this image-to-scene map have an exact mirror solution. For the aspect ratio we chose, no exact mirror solution exists. Yet, the errors in the viewing directions are small (see Fig. 8(c)). The caustic of this complex imaging system, shown in Fig. 8(d), was unknown until now.

\subsection{Skewed Plane Projection System}

We now present a catadioptric projection system geared towards avoiding occlusions. In typical frontal projection systems, the display surface is often occluded when the user comes between the projector and the display surface. Techniques to reduce occlusions [22] work to a limited extend and produce artifacts at the shadow edges.

We propose to minimize occlusions by positioning the projector very close to the display surface. However, in such a configuration, the image needs to be warped prior to projection. Digitally warping the image leads to image quality degradation due to re-sampling. Furthermore, projecting images onto large areas from such close proximity to the display surface is impossible using conventional projectors due to their restricted field of view. We therefore propose optically warping the image using catadioptrics.

Referring to Fig. 9 (a), let us assume that the display surface lies 1 foot below and 1 foot away from the projector and spans a $10^{\prime} \times 10^{\prime}$ square region. The projector's image plane is assumed to be parallel to the $X Y$-plane. The primary optics of the catadioptric projection system are assumed to be orthographic. The computed mirror shape using the weighted method, its associated scene projection errors and caustic are shown in Fig. 9 (b,c,d), respectively. As expected from the setup, the mirror is symmetric about a single plane. Note that, in spite of the projector being only a foot away from a large display surface, the errors in projection are negligible (less than 0.02 inches). 


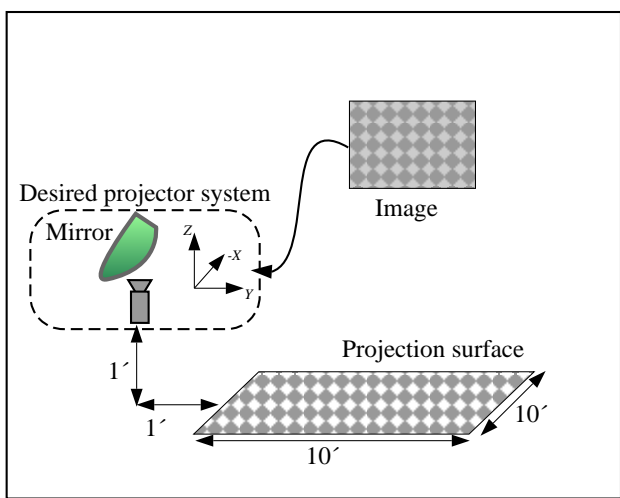

(a) Setup for projection system

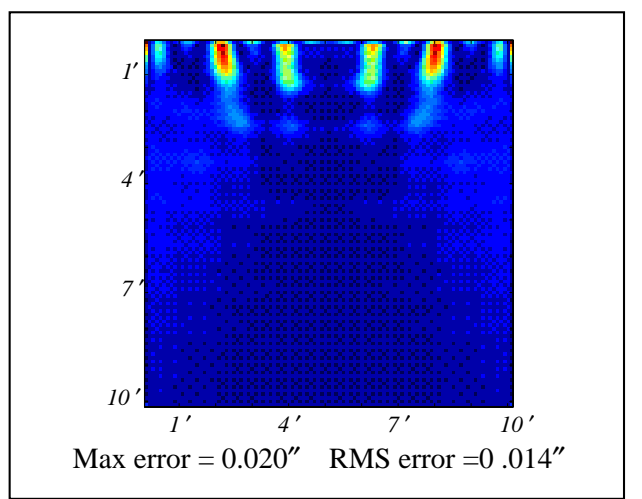

(c) Scene projection errors (in inches)

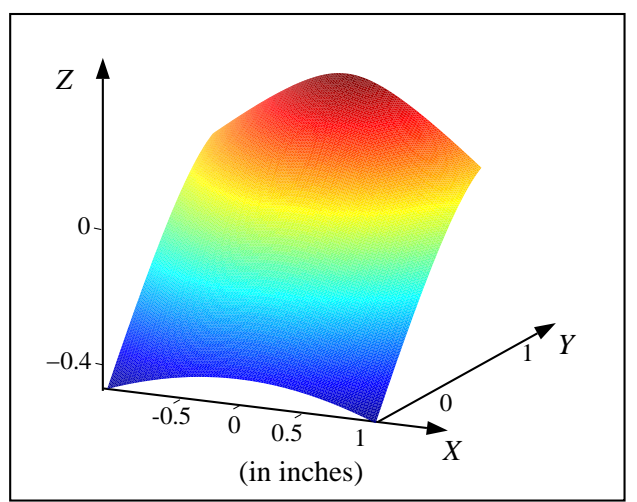

(b) Computed mirror in 3D

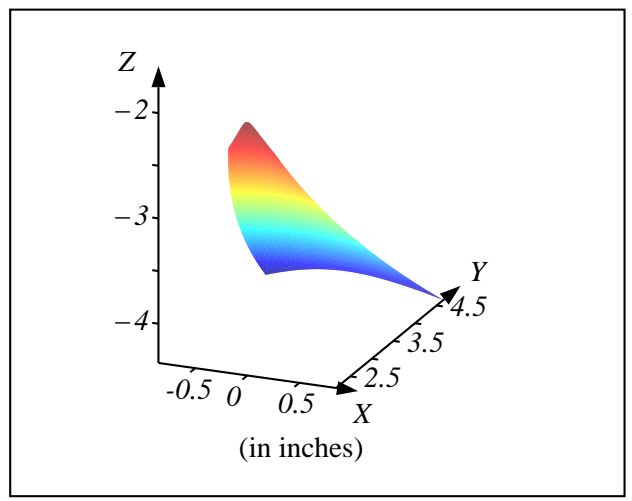

(d) Caustic of projection system

Figure 9: A novel catadioptric projection system designed using the proposed framework. We design a projection system that minimizes occlusions by placing it very close to the display surface. (a) The projector is located $1^{\prime}$ above and $1^{\prime}$ away from a $10^{\prime} \times 10^{\prime}$ display surface. (b) The computed threedimensional mirror shape. (c) Projection errors, measured on the display surface and are negligible $\left(<0.02^{\prime}\right)$. (d) The projection system does not have a single point of projection but rather a caustic, computed using the proposed framework.

\subsection{Conference Table Rectifying System}

We now present a novel catadioptric imaging system for video conferencing applications. Consider the scenario of imaging people seated around an elliptical conference table. We would like to acquire an image such that the curved edge of the table appears straight. Thus, all the people would appear as if seated at a straight desk.

We call such an imaging system as the conference table rectifying system. The setup of the mirror and table for this system are shown in Fig. 10(a). The table consists of a semi-circular section of radius 30 inches with two extended straight sides, each 30 inches long. The primary optics (telecentric lens) is assumed to be located roughly 30 inches behind this table facing away from 


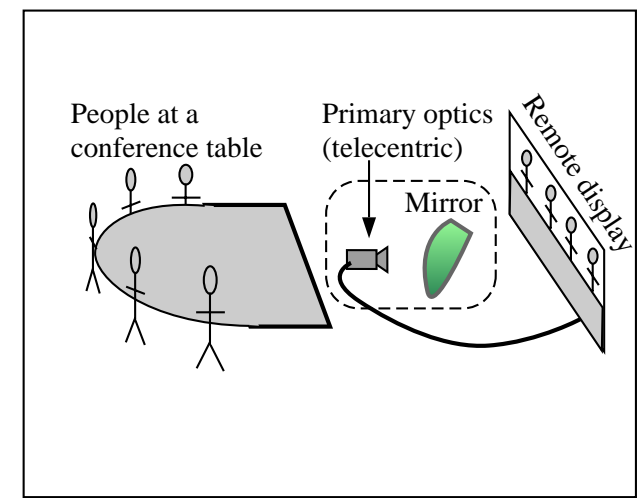

(a) Schematic of conference table rectifying system

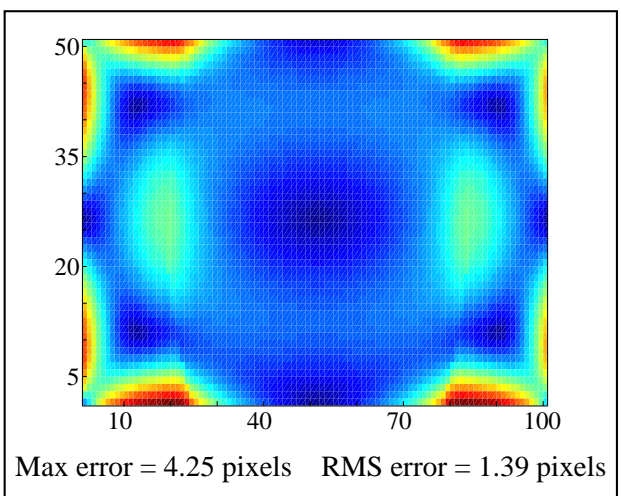

(c) Image projection errors (in pixels)

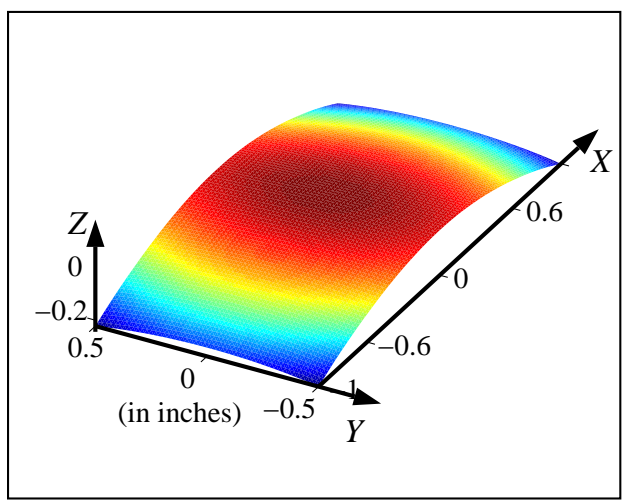

(b) Computed mirror in 3D

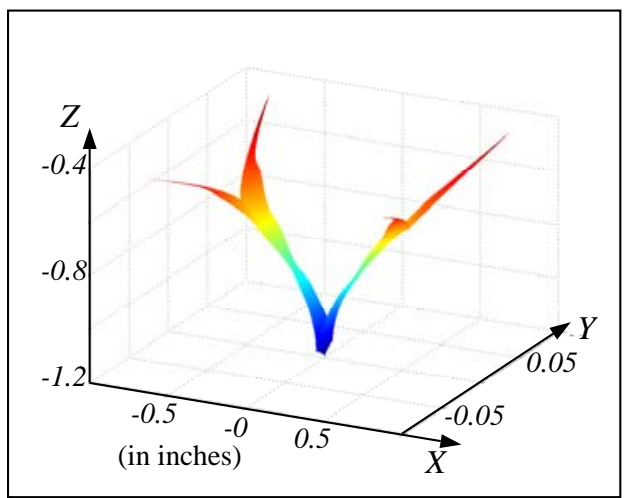

(d) Caustic of the imaging system

Figure 10: A new imaging system for video conferencing applications. (a) Setup of the imaging system designed to acquire images of people seated around a curved conference table as if seated along a straight bench. We use a telecentric lens based primary optics. (b) The computed "optimal" mirror shape in three dimensions. (c) Errors in the image projection are higher in this case as no mirror that performs the desired map exists. (d) The corresponding caustic surface is also computed. Since the mirror is flatter along one direction, the caustic is almost linear along that direction.

the scene into a mirror.

The computed mirror shape is shown in Fig. 10(b). Note that no mirror shape exists that provides the required image-to-scene map. The computed mirror shape approximates the desired mapping by minimizing image distortions. In this case, it was therefore important to use the modified image error based metric discussed in Section3. The resulting image errors are shown in Fig. 10(c). This imaging system also does not have a single viewpoint. Its caustic is shown in Fig. 10(d).

We also compare our method against an hypothetical ideal sensor and previous shape based methods. While the previous method[25] produces large image errors (Max. $=10.14$, RMS = 3.0) (see Fig. 10(e)), our approach drastically reduces errors by about 80\% (Max. = 1.92, RMS 


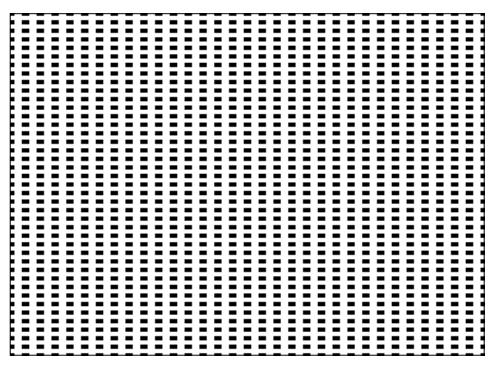

(a)

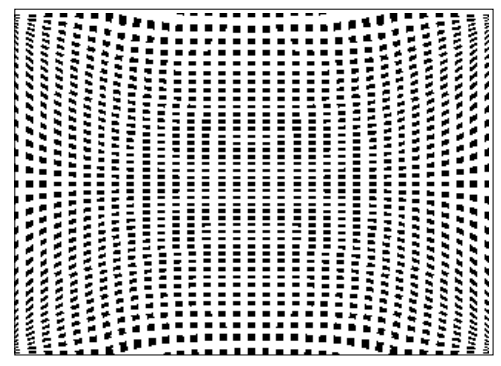

(b)

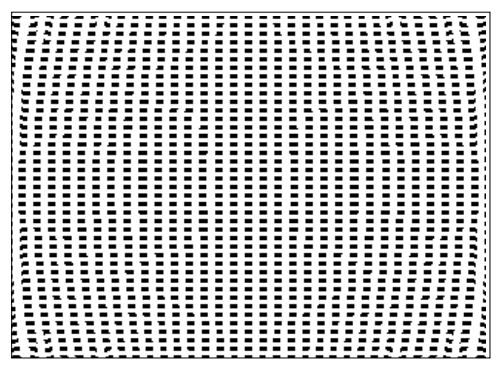

(c)

Figure 11: A checker board pattern acquired using (a) a hypothetically ideal system, (b) a mirror designed to minimize errors in orientation of the surface normals $[11,25]$, showing strong distortions (Max. = 10.14 , RMS $=3.0$ ) and $(\mathrm{c})$ our method that minimizes image errors (Max. $=1.92, \mathrm{RMS}=0.6$ ). Note that our method reduces errors by over $80 \%$.

=0.6). This is demonstrated by the large distortions visible in Fig. 11(b) the shape based spline approach, as opposed to Fig. 11(a) the ground truth or Fig. 11(c) our proposed approach.

\subsection{Spherical Projection System}

Our last example consists of a novel projection system for an immersive viewing environment. The setup consists of a spherical projection screen of radius $10^{\prime}$. The observer is located at the center of the sphere, while the projector is located directly above the observer at the pole of the sphere as in Fig.12(a). The goal is to project a section of a spherical panorama from the pole, as if projected from the center.

For a given field of view, Figs.12(b,c) illustrate the computed mirror shape and the corresponding scene errors. Also, we compare errors in projection due to earlier shape based approaches (Max $=12.65$, RMS $=4.8$ inches) shown in Fig.12(e) against that using our proposed scene error based method $(\operatorname{Max}=8.43$, RMS $=2.84)$ (see Fig.12(f)). Projection errors using our method were reduced by over $30 \%$.

A point to note is that the same method was used to estimate all the mirror shapes. We can compute single viewpoint as well as non-single viewpoint systems just as easily. This makes our method truly flexible and applicable to a large class of mirror design problems. 


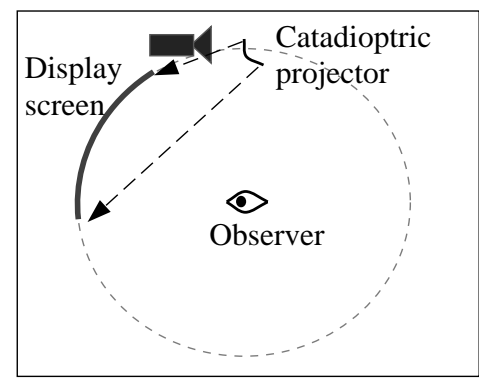

(a) Spherical projection system

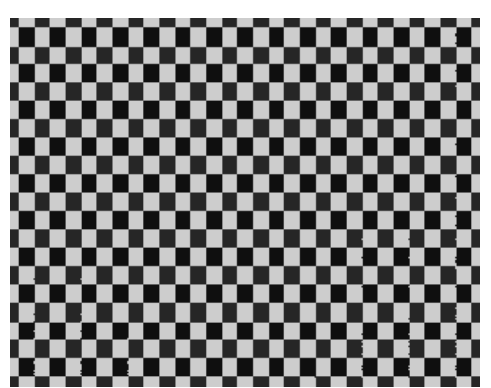

(d) Ideal checkerboard pattern

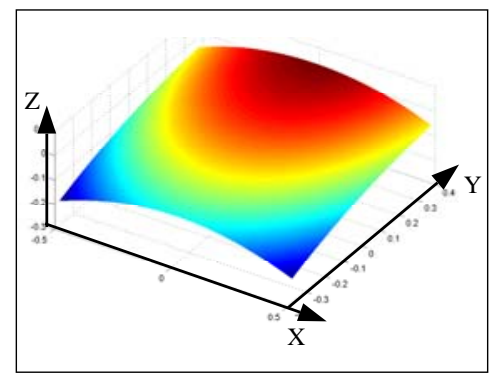

(b) Computed mirror in 3D

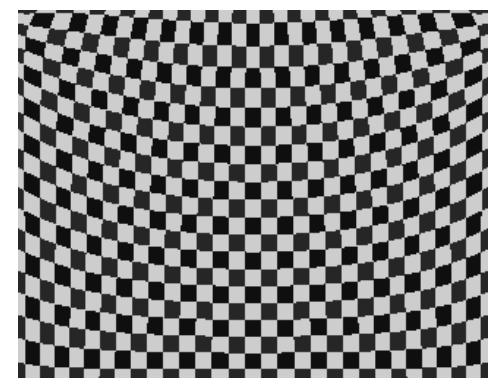

(e) Distorted pattern using previous[21] tool

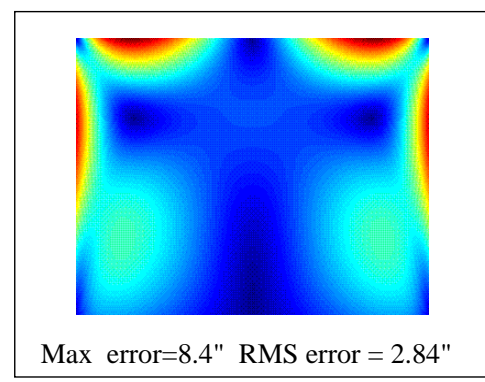

(c) Scene projection errors (inches)

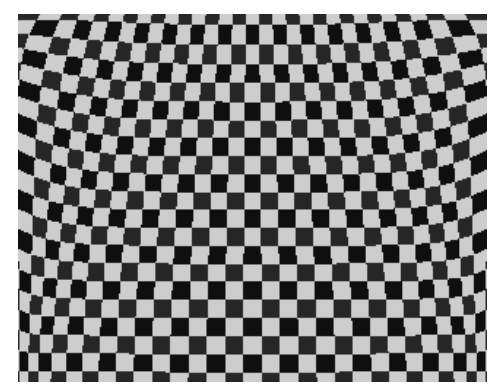

(f) Checkerboard with proposed method

Figure 12: (a) A spherical immersive screen with the observer seated in the center. The projector is located on the roof and is expected to project a section of a spherical panorama on the screen as shown. (b) The mirror computed using our scene projection error minimizing approach. (c) Errors in projection onto the sphere are very small in comparison with the mirror shape error minimizing technique. (d) Ideal checkerboard pattern projected on the sphere. (e) Equivalent checkerboard pattern using the mirror shape error minimizing method showing stronger distortions. (f) Checkerboard pattern projected using our proposed method is visibly much lesser distorted.

\section{Conclusions}

In this paper, we presented a framework to design general catadioptric imaging and projection systems. Such systems are assumed to consist of some known primary optics and an unknown mirror. Using our framework, the designer has complete freedom in designing arbitrary catadioptric systems. One can define any desired imaging geometry, by specifying a map from pixels in the image to points in the scene, which we called the image-to-scene map. Our method then automatically computes the optimal mirror shape that implements the desired image-to-scene map.

A major advantage of the method is that it can be used with all possible models for the primary optics. These include the perspective, orthographic and the generalized imaging model. Furthermore, the same method can be used to design a very large class of systems including rotationally 
symmetric, asymmetric, single viewpoint and non-single viewpoint systems. We also used our framework to compute the caustics of such general imaging systems.

We believe that the method we proposed is a powerful tool and throws wide open the space of projector and imaging systems that can be designed.

\section{Acknowledgements}

This research was supported by the DARPA HID Program under Contract No. N00014-00-1-0916.

\section{References}

[1] S. Baker and S. K. Nayar. A Theory of Catadioptric Image Formation. In Proc. ICCV, pages 35-42, 1998.

[2] R. Benosman, E. Deforas, and J. Devars. A New Catadioptric Sensor for the Panoramic Vision of Mobile Robots. In Proc. OMNIVIS, 2000.

[3] S. Bogner. Introduction to Panoramic Imaging. In IEEE SMC Conference, volume 54, pages 3100-3106, 1995.

[4] M. Born and E. Wolf. Principles of Optics. Permagon Press, 1965.

[5] A.M. Bruckstein and T.J. Richardson. Omniview Cameras with Curved Surface Mirrors. In Proc. OMNIVIS, pages 79-84, 2000.

[6] J. Chahl and M. Srinivasan. Reflective Surfaces for Panoramic Imaging. Applied Optics, 36(31):8275-8285, 1997.

[7] S. Derrien and K. Konolige. Aproximating a Single Viewpoint in Panoramic Imaging Devices. In International Conference on Robotics and Automation, pages 3932-3939, 2000.

[8] S. Gächter. Mirror Design for an Omnidirectinal Camera with a Uniform Cylindrical Projection when using the SVAVISCA Sensor. Technical Report CTU-CMP-2001-03, Czech Technical University, 2001.

[9] J Gaspar, C. Decco, J. Okamoto Jr, and J. Santos-Victor. Constant Resolution Omnidirectional Cameras. In Proc. OMNIVIS, page 27, 2002. 
[10] M. Grossberg and S.K. Nayar. A General Imaging Model and a Method for Finding its Parameters. In Proc. ICCV, pages 108-115, 2001.

[11] M.A. Halstead, B.A. Barsky, S.A. Klein, and R.B. Mandell. Reconstructing curved surfaces from specular reflection patterns using spline surface fitting of normals. In Proc. SIGGRAPH, pages $335-342,1996$.

[12] E. Hecht. OPTICS. Addison-Wesley, Massachusetts, second edition, 1987.

[13] A. Hicks. Differential Methods in Catadioptric Sensor Design with Applications to Panoramic Imaging. Technical report, Drexel University, Computer Science, 2002.

[14] R.A. Hicks and R. Bajcsy. Catadioptric Sensors that Approximate Wide-Angle Perspective Projections. In Proc. CVPR, pages I:545-551, 2000.

[15] R.A. Hicks and R.K. Perline. Geometric Distributions for Catadioptric Sensor Design. In Proc. CVPR, pages I:584-589, 2001.

[16] R.A Hicks and R.K Perline. Equi-areal Catadioptric Sensors. In Proc. OMNIVIS, page 13, 2002.

[17] F. M. Marchese and D. G. Sorrenti. Mirror Design of a Prescribed Accuracy Omni-directional Vision System. In Proc. OMNIVIS, page 136, 2002.

[18] V. Nalwa. A True Omnidirectional Viewer. Technical report, Bell Laboratories, Holmdel, NJ 07733, U.S.A., 1996.

[19] S. K. Nayar. Catadioptric Omnidirectional Cameras. In Proc. CVPR, pages 482-488, 1997.

[20] D. Rees. Panoramic Television viewing System. United States Patent No.3,505,465, 1970.

[21] M.V. Srinivasan. New Class of Mirrors for Wide-Angle Imaging. In Proc. OMNIVIS, 2003.

[22] R. Sukthankar, T.J. Cham, and G. Sukthankar. Dynamic Shadow Elimination for MultiProjector Displays. In Proc. CVPR, pages II:151-157, 2001.

[23] R. Swaminathan, M. D. Grossberg, and S. K. Nayar. Caustics of Catadioptric Cameras. In Proc. ICCV, pages II:2-9, 2001.

[24] R. Swaminathan, S. K. Nayar, and M. D. Grossberg. A General Framework to Design Catadioptric Imaging and Projection Systems. Technical Report CUCS-017-03, Columbia Univeristy, 2003. 
[25] R Swaminathan, S.K Nayar, and M.D. Grossberg. Framework for Designing Catadioptric Projection and Imaging Systems. In Proc. OMNIVIS, 2003.

[26] Y. Yagi and M. Yachida. Real-Time Generation of Environmental Map and Obstacle Avoidance Using Omnidirectional Image Sensor with Conic Mirror. In Proc. CVPR, pages 160-165, 1991.

[27] K. Yamazawa, Y. Yagi, and M. Yachida. Omnidirectional Imaging with Hyperboloidal Projection. In Proc. IEEE/RSJ International Conference on Intelligent Robots and Systems, pages 1029-1034, 1993. 